\title{
Accuracy of direct genomic values for functional traits in Brown Swiss cattle
}

\author{
M. Kramer, ${ }^{\star 1}$ M. Erbe, ${ }^{\star}$ F. R. Seefried,† B. Gredler, $†$ B. Bapst, $\uparrow$ A. Bieber,‡ and H. Simianer ${ }^{\star}$ \\ *Department of Animal Science, Animal Breeding and Genetics Group, Georg-August-Universität Göttingen, Albrecht-Thaer-Weg 3, \\ 37075 Göttingen, Germany \\ †Qualitas AG, Chamerstraße 56, 6300 Zug, Switzerland \\ $\neq F i B L-R e s e a r c h$ Institute of Organic Agriculture, Ackerstrasse 21, 5070 Frick, Switzerland
}

\section{ABSTRACT}

In this study, direct genomic values for the functional traits general temperament, milking temperament, aggressiveness, rank order in herd, milking speed, udder depth, position of labia, and days to first heat in Brown Swiss dairy cattle were estimated based on $\sim 777,000$ (777K) single nucleotide polymorphism (SNP) information from 1,126 animals. Accuracy of direct genomic values was assessed by a 5 -fold cross-validation with 10 replicates. Correlations between deregressed proofs and direct genomic values were 0.63 for general temperament, 0.73 for milking temperament, 0.69 for aggressiveness, 0.65 for rank order in herd, 0.69 for milking speed, 0.71 for udder depth, 0.66 for position of labia, and 0.74 for days to first heat. Using the information of $\sim 54,000$ (54K) SNP led to only marginal deviations in the observed accuracy. Trying to predict the $20 \%$ youngest bulls led to correlations of $0.55,0.77,0.73$, $0.55,0.64,0.59,0.67$, and 0.77 , respectively, for the traits listed above. Using a novel method to estimate the accuracy of a direct genomic value (defined as correlation between direct genomic value and true breeding value and accounting for the correlation between direct genomic values and conventional breeding values) revealed accuracies of $0.37,0.20,0.19,0.27,0.48,0.45$, 0.36 , and 0.12 , respectively, for the traits listed above. These values are much smaller but probably also more realistic than accuracies based on correlations, given the heritabilities and samples sizes in this study. Annotation of the largest estimated SNP effects revealed 2 candidate genes affecting the traits general temperament and days to first heat.

Key words: direct genomic value prediction, accuracy of direct genomic value, functional trait, gene annotation

Received May 23, 2013.

Accepted November 25, 2013.

${ }^{1}$ Corresponding author: mkramer@gwdg.de

\section{INTRODUCTION}

Genomic selection is well established in dairy cattle breeding, and accuracies of direct genomic values (DGV) for traditional production or conformation traits are high (e.g., Segelke et al., 2012). To maximize the benefit, genomic selection should also be used for scarcely recorded functional traits, which are becoming increasingly relevant in dairy cattle breeding. For this reason, one aim of our study was to apply the estimation of DGV to a set of functional traits. High-density chips with information of more than $777,000(\mathbf{7 7 7 K})$ SNP are increasingly available in cattle breeding. We thus compared results from an estimation of DGV with $777 \mathrm{~K}$ data with the results of estimation of DGV with $\sim 54,000(\mathbf{5 4 K})$ SNP data. To assess the accuracy of DGV, it is easy to calculate the correlation between DGV and conventional breeding value (EBV), but this correlation is different from the correlation between DGV and true breeding value (TBV), which is normally used in practical breeding to reflect the accuracy of a breeding value. To account for this, the correlation between DGV and EBV is often divided by the accuracy of the EBV. As stated by Amer and Banos (2010), this correction leads to an overestimation of accuracy; therefore, we applied a new method proposed by Wellmann et al. (2013) to estimate more realistic accuracies. To date, only few genome-wide association studies for functional traits have been conducted in Brown Swiss (BS) cattle (e.g., Guo et al., 2012). Another aim of our work was thus to screen the genome for SNP with large effects on the observed traits to find genes that are associated with these SNP and could influence the respective trait.

\section{MATERIALS AND METHODS}

\section{Genotypic Data}

The $777 \mathrm{~K}$ genotypes used in this study were derived by imputation from a data set of $880 \mathrm{BS}$ animals genotyped with the Illumina BovineHD chip (727 cows, 153 bulls) and from a data set of 6,016 animals genotyped with the Illumina Bovine SNP50 chip (548 
cows, 5,468 bulls; both chips from Illumina Inc., San Diego, CA). Before imputation, both data sets were checked for quality separately: SNP with minor allele frequency $<0.5 \%$, a call rate $<90 \%$, missing position, or position on the sex chromosomes were excluded. In addition, within the $777 \mathrm{~K}$ data set, mitochondrial SNP and 1 SNP of each of 55 pairs of SNP with identical position but different denomination, respectively, were excluded. Animals with a call rate $<90 \%$ were excluded from both data sets. After quality checks, imputation was done by using FImpute (Sargolzaei et al., 2011) from 39,004 to $627,306 \mathrm{SNP}$, using a combination of family and population imputation. This method led to a correlation of 0.99 between true genotype and imputed genotype if both parents were genotyped with the $777 \mathrm{~K}$ chip and to a correlation of 0.97 between true genotype and imputed genotype if no close relatives were genotyped with the $777 \mathrm{~K}$ chip (Gredler et al., 2013). We used a subset of 1,126 animals (930 cows from Switzerland with phenotype information for the traits observed and 196 bulls with performance records of at least one daughter) for estimation of DGV and SNP effects in our study.

\section{Phenotypic Data}

Deregressed proofs (DRPF) for the traits general temperament, milking temperament, aggressiveness, rank order in herd, milking speed, udder depth, position of labia, and days to first heat of the 1,126 BS animals were used as quasi phenotypes. For detailed information on the trait definitions, see Kramer et al. (2013). Although some of these traits are routinely evaluated and multiple-trait across-country evaluation (MACE) proofs would have been available, we decided to use our own breeding values to evaluate the possibilities of genomic estimation based on genotypes and phenotypes of a mixed data set of cows and bulls. The DRPF for individual $i$ within a given trait were derived as described in Garrick et al. (2009):

$$
D R P F_{i}=\frac{E B V_{i}}{r_{E B V i}^{2}}
$$

where $D R P F_{i}$ is the DRPF of individual $i, E B V_{i}$ is the EBV for individual $i$, and $r_{E B V}^{2}$ is the squared accuracy of the $E B V_{i}$ for individual $i$. In our study, EBV of cows with several phenotypic measurements as well as EBV of bulls with a different number of daughters (mean: 3.22; range: 1-31) were used. Distribution of birth year of animals is shown in Figure 1. Ninety-five percent of bulls were born between the years 1988 and 2004, and $95 \%$ of the cows were born between the years 1998 and 2007. Accuracies of conventional breeding values $\left(r_{E B V}\right)$ were in a wide range and, on average, higher in cows than in bulls for most traits (Table 1).

\section{Model}

The DGV were estimated using the model $\mathbf{y}=\mathbf{1} \mu+$ $\mathbf{Z g}+\mathbf{e}$, where $\mathbf{y}$ is a vector of DRPF, $\mathbf{1}$ is a vector of $1 \mathrm{~s}, \mu$ is the overall mean, $\mathbf{g}$ is a vector of additive genetic effects, $\mathbf{e}$ is a vector of residual effects, and $\mathbf{Z}$ is a design matrix allocating genetic effects to the observations. For random elements, the distributions $\mathbf{e} \sim N\left(0, \mathbf{R} \sigma_{e}^{2}\right)$ and $\mathbf{g} \sim N\left(0, \mathbf{G} \sigma_{g}^{2}\right)$ were assumed, where $\mathbf{R}$ is a diagonal matrix with $1 / w_{i}$ on the diagonal and $w_{i}$ is a weighting factor for the residual variance, and $\mathbf{G}$ is a genomic relationship matrix. Additive genetic variance

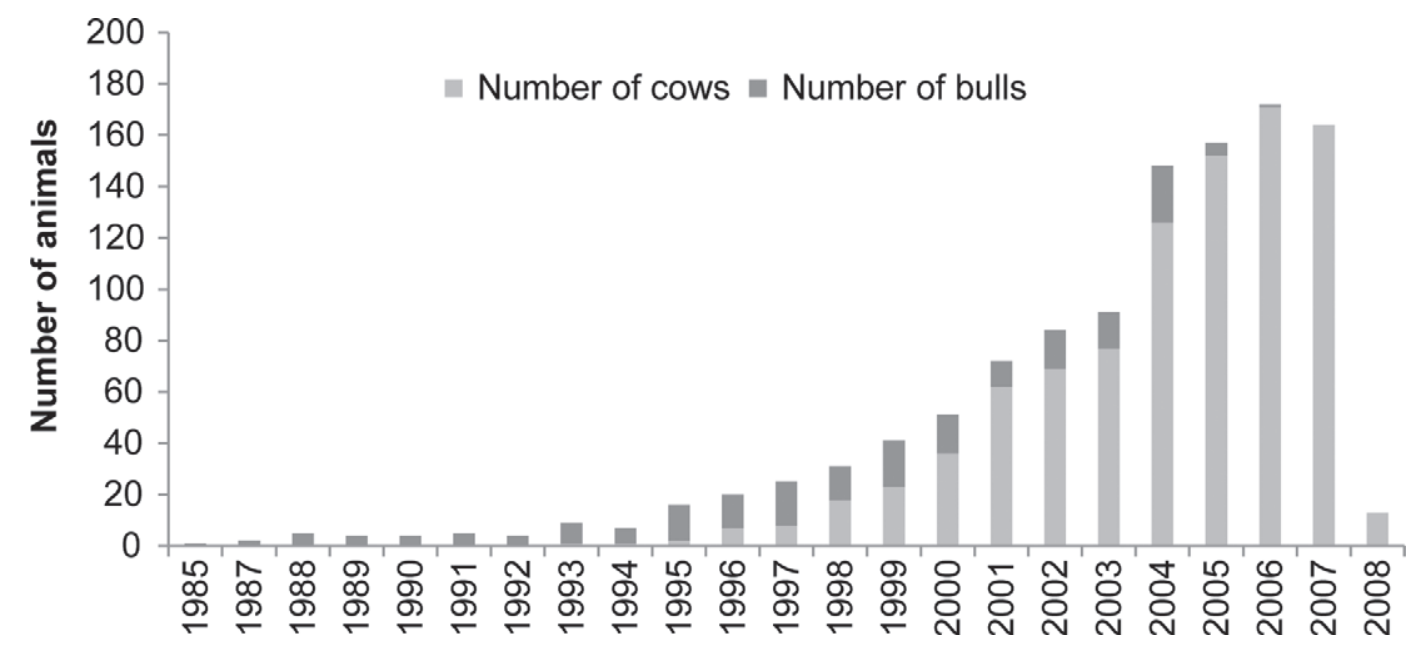

Figure 1. Distribution of birth year for genotyped cows and bulls. 
Table 1. Heritabilities $\left(h^{2}\right)$ with standard error (SE), mean accuracies $\left(r_{E B V}\right)$ with standard deviation (SD) of estimated or conventional breeding values (EBV) and number of records used for estimation of EBV (n EBV) for the different groups of animals for the traits observed

\begin{tabular}{lccccc}
\hline & & \multicolumn{3}{c}{$r_{E B V} \pm \mathrm{SD}$} & \\
\cline { 3 - 5 } & $h^{2} \pm \mathrm{SE}$ & $\begin{array}{c}\text { All animals } \\
(\mathrm{n}=1,126)\end{array}$ & $\begin{array}{c}\text { Cows } \\
(\mathrm{n}=930)\end{array}$ & $\begin{array}{c}\text { Bulls } \\
(\mathrm{n}=196)\end{array}$ & $\mathrm{n}$ EBV \\
\hline Trait & $0.38 \pm 0.07$ & $0.66 \pm 0.08$ & $0.68 \pm 0.05$ & $0.59 \pm 0.12$ & 2,312 \\
General temperament & $0.04 \pm 0.04$ & $0.30 \pm 0.06$ & $0.30 \pm 0.05$ & $0.29 \pm 0.09$ & 2,259 \\
Milking temperament & $0.12 \pm 0.08$ & $0.34 \pm 0.08$ & $0.34 \pm 0.08$ & $0.33 \pm 0.10$ & 2,309 \\
Aggressiveness & $0.16 \pm 0.06$ & $0.51 \pm 0.07$ & $0.51 \pm 0.05$ & $0.47 \pm 0.12$ & 2,304 \\
Rank order in herd & $0.42 \pm 0.06$ & $0.72 \pm 0.08$ & $0.74 \pm 0.05$ & $0.62 \pm 0.12$ & 4,540 \\
Milking speed & $0.42 \pm 0.06$ & $0.66 \pm 0.08$ & $0.67 \pm 0.06$ & $0.58 \pm 0.12$ & 2,195 \\
Udder depth & $0.28 \pm 0.06$ & $0.61 \pm 0.08$ & $0.62 \pm 0.07$ & $0.54 \pm 0.12$ & 2,232 \\
Position of labia & $0.02 \pm 0.04$ & $0.18 \pm 0.04$ & $0.19 \pm 0.04$ & $0.18 \pm 0.06$ & 1,678 \\
Days to first heat & & & & &
\end{tabular}

$\left(\sigma_{g}^{2}\right)$ and residual variance $\left(\sigma_{e}^{2}\right)$ were estimated from the complete data set using ASReml (Gilmour et al., 2009).

Because of the wide range of $r_{E B V}$, residual errors were also in a wide range and it was thus necessary to account for different residual variances. The weighting factor for the reciprocal of the residual error of individual $i\left(w_{i}\right)$ was calculated as described in Garrick et al. (2009):

$$
w_{i}=\frac{1-h^{2}}{h^{2}\left(c+\frac{1-r_{E B V_{i}}^{2}}{r_{E B V_{i}}^{2}}\right)},
$$

where $h^{2}$ is the heritability of the observed trait, $c$ is the fraction of genetic variance that is not explained by markers, and $r_{E B V_{i}}^{2}$ is the squared accuracy of the EBV. We assumed that the complete genetic variance was explained by the $627 \mathrm{k}$ SNP markers and so $c$ was set to 0 . This assumption is also made in routine breeding value estimation for functional traits in Simmental cattle in Germany and Austria (Edel et al., 2011).

The genomic relationship matrix $\mathbf{G}$ was set up from the SNP data as described in VanRaden (2008):

$$
\mathbf{G}=\frac{(\mathbf{M}-\mathbf{P})(\mathbf{M}-\mathbf{P})^{\prime}}{2 \sum_{j=1}^{m} p_{j}\left(1-p_{j}\right)},
$$

where $p_{j}$ is the frequency of the second allele at locus $j ; m$ is the total number of SNP; $\mathbf{M}$ is a matrix with genotypes coded as 0,1 , and 2 in columns and animals in rows; and $\mathbf{P}$ is a matrix with all elements in column $j$ being $2 p_{j}$.

The correlation between DGV and DRPF $\left(\boldsymbol{r}_{D G V, D R P F}\right)$ was assessed by random 5 -fold cross-validation with 10 replicates and was calculated as an average over all 50 replicates of predicted folds.

To study the influence of the age structure, we also performed one cross-validation run with 777K SNP information with animals sorted by age, where the DGV of the youngest 177 animals from birth year 2007-2008 (approximately one-fifth) were predicted with use of the older animals (birth years 1985-2006).

Random cross-validation was further carried out with the $54 \mathrm{~K}$ SNP panel. For cross-validation with $54 \mathrm{~K}$ information, the data set was restricted to the 39,004 SNP from the 54K SNP chip after filtering, and $\sigma_{g}^{2}$ and $\sigma_{e}^{2}$ were estimated again using a $\mathbf{G}$ matrix based on $54 \mathrm{~K}$ information only.

The SNP effects were derived for the 777K SNP data as described in, for example, Ober et al. (2012):

$$
\hat{\mathbf{s}}=\frac{\hat{\sigma}_{g}^{2}}{2 \sum_{j=1}^{m} p_{j}\left(1-p_{j}\right)}(\mathbf{M}-\mathbf{P})^{\prime}\left(\hat{\sigma}_{g}^{2} \mathbf{G}+\hat{\sigma}_{e}^{2} \mathbf{R}\right)^{-1}(\mathbf{y}-\mathbf{1} \hat{\mu}),
$$

where $\hat{\mathbf{s}}$ is a vector of SNP effects, and all other factors are as described above. The SNP effects were then standardized as follows:

$$
\hat{\mathbf{s}}_{S T D}=\frac{\hat{\mathbf{s}}}{\sqrt{\hat{\sigma}_{S N P}^{2}}}
$$

with

$$
\hat{\sigma}_{S N P}^{2}=\frac{\hat{\sigma}_{g}^{2}}{2 \sum_{j=1}^{m} p_{j}\left(1-p_{j}\right)},
$$

where $\hat{\mathbf{s}}_{S T D}$ is a vector of standardized SNP effects and all other factors are described above. The SNP with an 
absolute standardized effect $>4 \mathrm{SD}$ were considered SNP with a high influence on the specific trait. In the Manhattan plots (not shown), some distinct peaks formed by the high-influence SNP were obvious. All genes up to $100 \mathrm{~kb}$ upstream and downstream of the SNP position with the most extreme value within these peaks of high influence SNP were annotated using the map viewer option of the bovine genome sequence assembly (Bos taurus 6.1; http://www.ncbi.nlm.nih.gov/ projects/mapview/map_search.cgi?taxid=9913\&build $=6.1$ ) to find genes that might causatively influence the respective trait.

The correlation $r_{D G V, D R P F}$ is different from the correlation between DGV and TBV, which is the accuracy of genomic breeding values $\left(r_{D G V}\right)$. Note that the often reported reliability of genomic breeding values is the square of the accuracy $\left(r_{D G V}^{2}\right)$ used in our study. Often, $r_{D G V}$ is estimated as $r_{D G V, D R P F} / r_{E B V}$, where it is further assumed that $r_{D G V, D R P F}=r_{D G V, E B V}$. It was noted by Amer and Banos (2010) that with this approach an overlap of testing and training sets in cross-validation may lead to an overestimation of $r_{D G V}$. Wellmann et al. (2013) suggested correcting for this bias by fitting the model

$$
\hat{r}_{D G V}=r_{D G V, D R P F}-\hat{a}_{1}\left(r_{E B V}^{V S}-1\right),
$$

where $\hat{r}_{D G V}$ is the estimated accuracy of DGV defined as correlation between DGV and TBV, $r_{D G V . D R P F}$ is the estimated correlation between DGV and DRPF in 1 fold of a cross-validation, $r_{E B V}^{V S}$ is the mean accuracy of EBV in the validation set of this fold, and $\hat{a}_{1}$ is a regression coefficient estimated from the equation

$$
r_{D G V, D R P F}=a_{0}+a_{1} r_{E B V}^{V S}+a_{2} r_{E B V}^{T S}+e,
$$

where $a_{0}, a_{1}$, and $a_{2}$ are the intercept and fixed regression coefficients, $e$ is the residual error, and $r_{E B V}^{T S}$ is the mean accuracy of EBV in the training set of the specific fold. For further details, see Wellmann et al. (2013).

For this approach, data sets with different accuracies of conventional breeding values $\left(r_{E B V}\right)$ are required to fit the regression model. As described in Wellmann et al. (2013), we performed this by fitting Eq. [2] for all traits simultaneously.

If the accuracy of DGV is compared with the accuracy of EBV, EBV should be based only on pedigree information because this is also available in an early stage of an animal's life. We thus approximated accuracies of EBV based on pedigree information from the average accuracies of EBV of bulls and cows (Table 1) by using the formula

$$
r=\sqrt{0.25\left(r_{S}^{2}+r_{D}^{2}\right)},
$$

where $r_{S}^{2}$ is the reliability of the sire's EBV and $r_{D}^{2}$ is the reliability of the dam's EBV. This formula is also used by, for example, Edel et al. (2010).

\section{RESULTS AND DISCUSSION}

\section{Correlation Between DGV and DRPF}

The values for $r_{D G V . D R P F}$ from 5 -fold random crossvalidation with 10 replicates with $777 \mathrm{~K}$ information are shown in Figure 2. With random cross-validation, we obtained $r_{D G V, D R P F}( \pm \mathrm{SD})$ of $0.63 \pm 0.05$ for general temperament, $0.73 \pm 0.04$ for milking temperament, $0.69 \pm 0.06$ for aggressiveness, $0.65 \pm 0.04$ for rank order in herd, $0.69 \pm 0.03$ for milking speed, $0.71 \pm$ 0.03 for udder depth, $0.66 \pm 0.03$ for position of labia, and $0.74 \pm 0.02$ for days to first heat between DGV and DRPF. The correlations between DGV and DRPF after stratification by age were in a range of 0.55 and 0.77 and thus were slightly lower, on average, than correlations from random cross-validation (Figure 2). The $r_{D G V, D R P F}$ values for the youngest animals deviated from the average values from random cross-validation between +0.04 for milking temperament and aggressiveness and -0.12 for udder depth, but were mostly in the range of values obtained by random cross-validation. These results are in line with Saatchi et al. (2011), who compared random cross-validation and cross-validation by age with 16 traits of American Angus beef cattle and also found higher accuracy for most traits with random cross-validation compared with stratification by age. If dairy cattle breeding values are estimated in practice, information from older animals (EBV of bulls with a certain number of daughters in milk or phenotypic measurements of lactating cows) is used to predict DGV for young animals; for example, bull calves for purchase by AI organizations. Thus, the age-stratified approach reflects the practical relevant case. However, due to the lack of resampling, the point estimate obtained for $r_{D G V, D R P F}$ in the age-stratified scenario may have a large stochastic error and should be interpreted with caution. Erbe et al. (2011) have suggested an age-stratification with resampling, which provides a distribution of estimates for the accuracy; however, this approach could not be implemented here due to the limited sample size. 


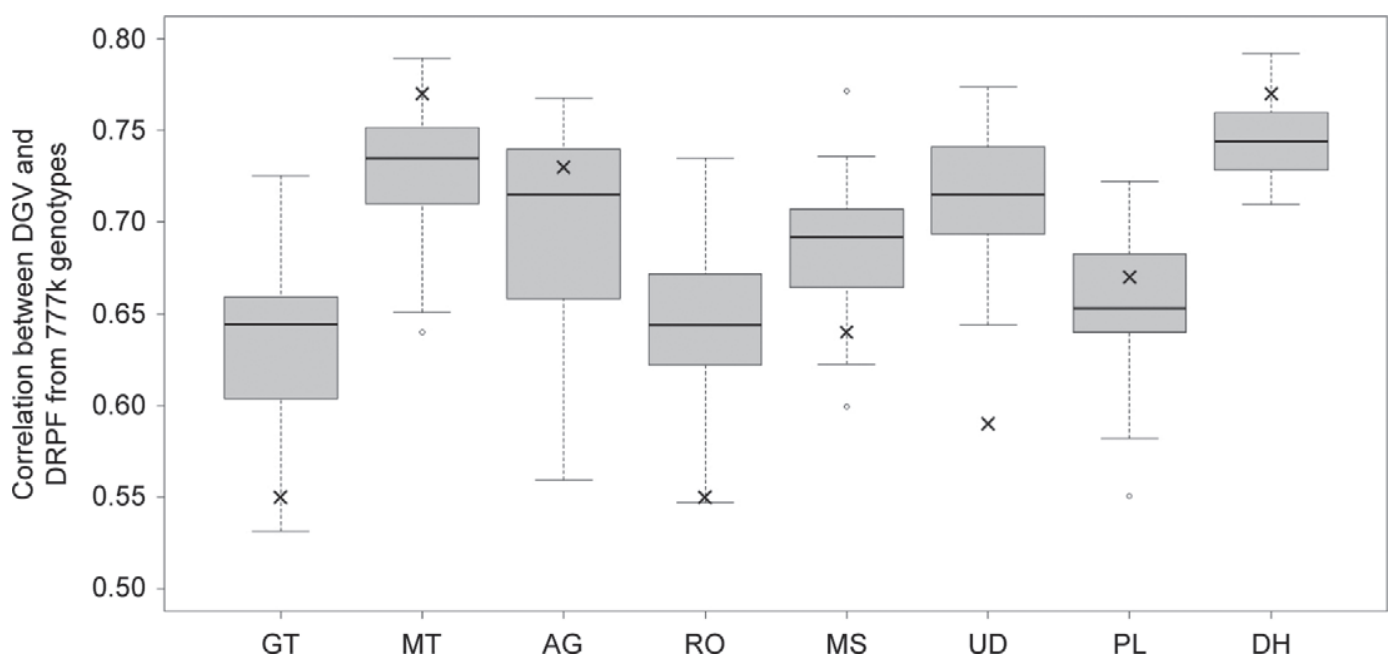

Figure 2. Correlations between direct genomic values (DGV) and deregressed proofs (DRPF) from cross-validation for general temperament (GT), milking temperament (MT), aggressiveness (AG), rank order in herd (RO), milking speed (MS), udder depth (UD), position of labia $(\mathrm{PL})$, and days to first heat $(\mathrm{DH})$, based on $777 \mathrm{~K}(\sim 777,000)$ SNP information. Results from 5 -fold random cross-validation with 10 replicates are indicated by boxes; results from stratification by age are indicated by $\times$.

In general, $r_{D G V, D R P F}$ values in our study were high, especially if the small number of animals in the data set $(\mathrm{n}=1,126)$ and the low $r_{E B V}(0.18-0.72)$ are taken into account. Erbe et al. (2012) found correlations between genomic breeding values and daughter trait deviations of $0.58,0.58$, and 0.56 for the traits milk yield, fat yield, and protein yield, respectively, in a data set of 2,257 Australian Holstein bulls. Thus, we reached apparently higher correlations between DGV and DRPF although our data set was smaller and $r_{E B V}$ was lower. This might be due to overestimation of accuracy of DGV, which will be discussed later.

Using $54 \mathrm{~K}$ genotype information (Figure 3 ), $r_{D G V, D R P F}$ were in a range between $0.74 \pm 0.02$ (days to first heat) and $0.63 \pm 0.05$ (general temperament) and thus show a difference from results from cross-validation with $777 \mathrm{~K}$ genotype information of $<0.01$ for all traits. This finding is in line with results from Su et al. (2012) with Nordic Holstein and Red Dairy cattle. The authors found only slight differences in reliability for DGV between $54 \mathrm{~K}$ genotype data and $777 \mathrm{~K}$ genotype data of up to 0.03 for the traits protein, fertility, and udder health. Our results were also in line with results from Erbe et al. (2012), who found correlations between genomic breeding value and daughter trait deviation from $54 \mathrm{~K}$ data being 0.01 to 0.03 less than those obtained with $777 \mathrm{~K}$ data in Australian Holstein cattle. This indicates that marker density of the 54K SNP chip appears sufficient and using the more expensive 777K SNP chip provides little improvement in predictive ability. This finding is further supported by, for example, VanRaden et al. (2013), who found that the high-density SNP chip provides only slightly better results. Those authors estimated a reliability of $61.1 \%$ when using the highdensity SNP chip, and a reliability of $60.7 \%$ when using a $54 \mathrm{~K}$ SNP chip, both as an average of 28 different traits.

\section{Accuracy of DGV}

The frequently used approach for calculating the accuracy of DGV as $r_{D G V, D R P F} / r_{E B V}$ would have resulted in accuracies $>1.0$ for most of the traits in our data set, which was characterized by high values of $r_{D G V \text {,DRPF }}$ and low but highly variable values for $r_{E B V}$ (Table 2). This is a clear case of overestimation of accuracy, as described by Amer and Banos (2010). We thus applied the approach of Wellmann et al. (2013) for estimation of $r_{D G V}$. Values of $r_{D G V}$ for the traits observed are shown in Table 2. The results indicate that it is generally possible to implement a system of genomic breeding value estimation for functional traits based on genotypes of phenotyped cows and sires with phenotyped daughters. Unfortunately, the limited number of individuals in our study was insufficient to achieve a large advantage of genomic prediction over parent average $\left(r_{P A}\right)$. Further work is needed to evaluate how many individuals have to be genotyped and phenotyped to achieve higher accuracy of GBV.

Our results indicate that $r_{D G V}$ and $r_{P A}$ are functions of heritability and size of the training set for the respective trait. Milking speed had the highest heritability and the largest training set; the highest $r_{D G V}$ and $r_{P A}$ were obtained for this trait (Table 2). In contrast, days 
Table 2. Correlation between direct genomic value and deregressed proof $\left(r_{D G V . D R P F}\right)$, heritability $\left(h^{2}\right)$, size of the training set (n EBV), accuracy of conventional breeding values from parent average $\left(r_{P A}\right)$, accuracy of genomic breeding values following the common approach $\left(r_{D G V, D R P F} / r_{E B V}\right)$, and accuracy of direct genomic values $\left(r_{D G V}\right)$ from application of the formula by Wellmann et al. (2013) for the traits observed

\begin{tabular}{lcccccc}
\hline Trait & $r_{D G V, D R P F}$ & $h^{2}$ & $\mathrm{n} \mathrm{EBV}$ & $r_{P A}$ & $r_{D G V, D R P F} / r_{E B V}$ & $r_{D G V}$ \\
\hline General temperament & 0.63 & 0.38 & 2,312 & 0.45 & 0.95 & 0.37 \\
Milking temperament & 0.73 & 0.04 & 2,259 & 0.21 & $>1$ & 0.20 \\
Aggressiveness & 0.69 & 0.12 & 2,309 & 0.24 & $>1$ & 0.19 \\
Rank order in herd & 0.65 & 0.16 & 2,304 & 0.35 & $>1$ & 0.27 \\
Milking speed & 0.69 & 0.42 & 4,540 & 0.48 & 0.96 & 0.48 \\
Udder depth & 0.71 & 0.42 & 2,195 & 0.44 & $>1$ & 0.45 \\
Position of labia & 0.66 & 0.28 & 2,232 & 0.41 & $>1$ & 0.36 \\
Days to first heat & 0.74 & 0.02 & 1,678 & 0.13 & $>1$ & 0.12 \\
\hline
\end{tabular}

to first heat had the smallest $r_{D G V}$, the smallest $r_{P A}$, the smallest training set, and also the smallest heritability (Table 2). In general, computing the observed accuracy of PA can be very helpful; however, most cross-validation studies do not test the parent average predictions directly, whereas validations using time truncation do.

As can be seen in Figure 4, $r_{D G V, D R P F}$ varied strongly within trait, whereas mean $r_{E B V}$ in the validation sets was trait-specifically quite consistent (from $r_{E B V} \approx 0.20$ for days to first heat to $r_{E B V} \approx 0.70$ for milking speed) across the samples. Fitting Eq. [2] to these data resulted in $a_{0}=0.75 \pm 0.01, a_{1}=-0.76 \pm 0.46$, and $a_{2}$ $=0.64 \pm 0.46$. Despite the large standard errors of the regression coefficients, our results were in line with the general findings of Wellmann et al. (2013) in another species (pigs); namely, that $a_{2}$ is positive and $a_{1}$ is negative. This latter result indicates that the accuracy of genomic breeding values, estimated as $r_{D G V, D R P F} / r_{E B V}$, is more biased upward, the smaller the accuracy of conventional breeding values $r_{E B V}$ is for a given trait.
By shifting the estimates of accuracy from $>0.9$ to 0.12 (for days to first heat) to 0.48 (for milking speed), the applied correction provides more realistic estimates of the realized accuracy (Table 2 ).

As mentioned above, standard errors of the estimates for $a_{0}, a_{1}$, and $a_{2}$ were high. One way to prevent these high standard errors might be to split the data set in a nonrandom way into training and validation sets; for example, cross validation by age. This might be an option for larger data sets (e.g., Erbe et al., 2011) but in our limited data set, only one replicate would be possible. Estimates from a regression based on these values will also have large standard errors. This strategy of splitting the data thus has no advantages in our data. We further tried to reduce the standard error by including random effects for trait and cross validation fold in Eq. [2] to estimate $a_{0}, a_{1}$, and $a_{2}$. This led to the same values for $a_{0}, a_{1}$, and $a_{2}$ and additionally to a slight increase of the standard error of $a_{0}$ as well as a slight decreases of the standard errors for $a_{1}$ and $a_{2}$

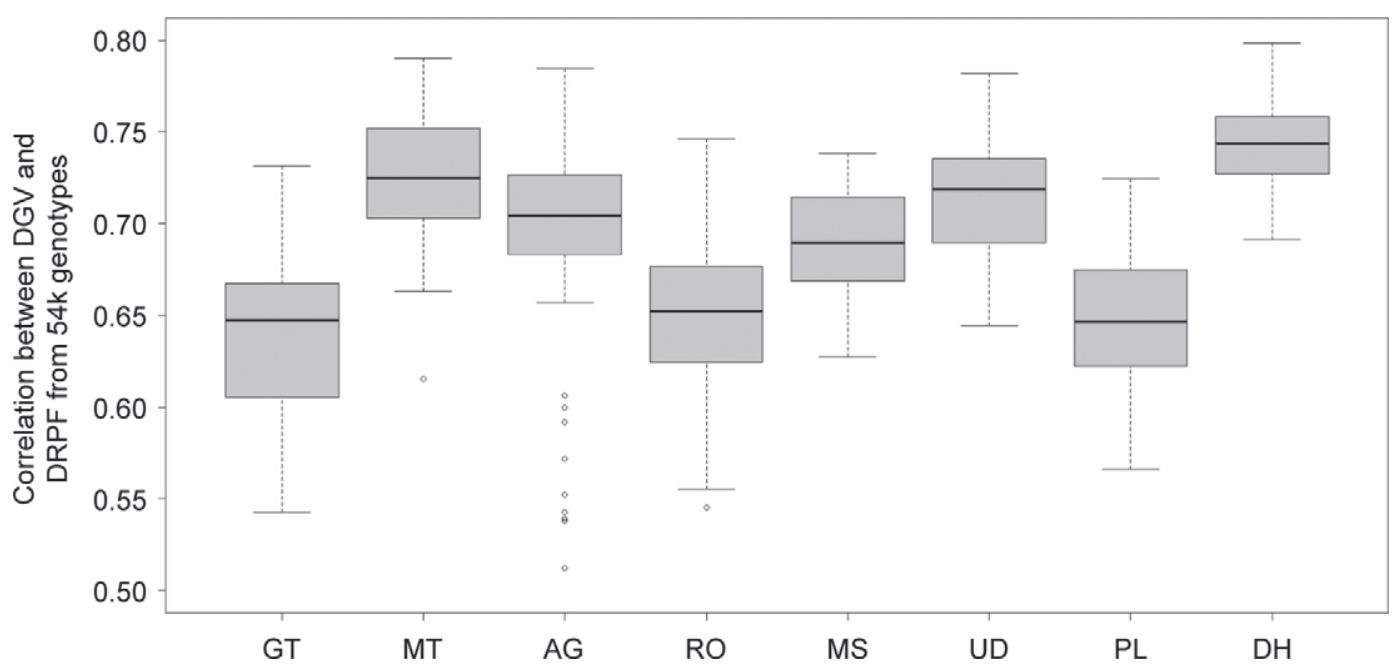

Figure 3. Correlations between direct genomic values (DGV) and deregressed proofs (DRPF) from cross-validation for general temperament (GT), milking temperament (MT), aggressiveness (AG), rank order in herd (RO), milking speed (MS), udder depth (UD), position of labia (PL), and days to first heat $(\mathrm{DH})$, based on $54 \mathrm{~K}(\sim 54,000)$ SNP information. 


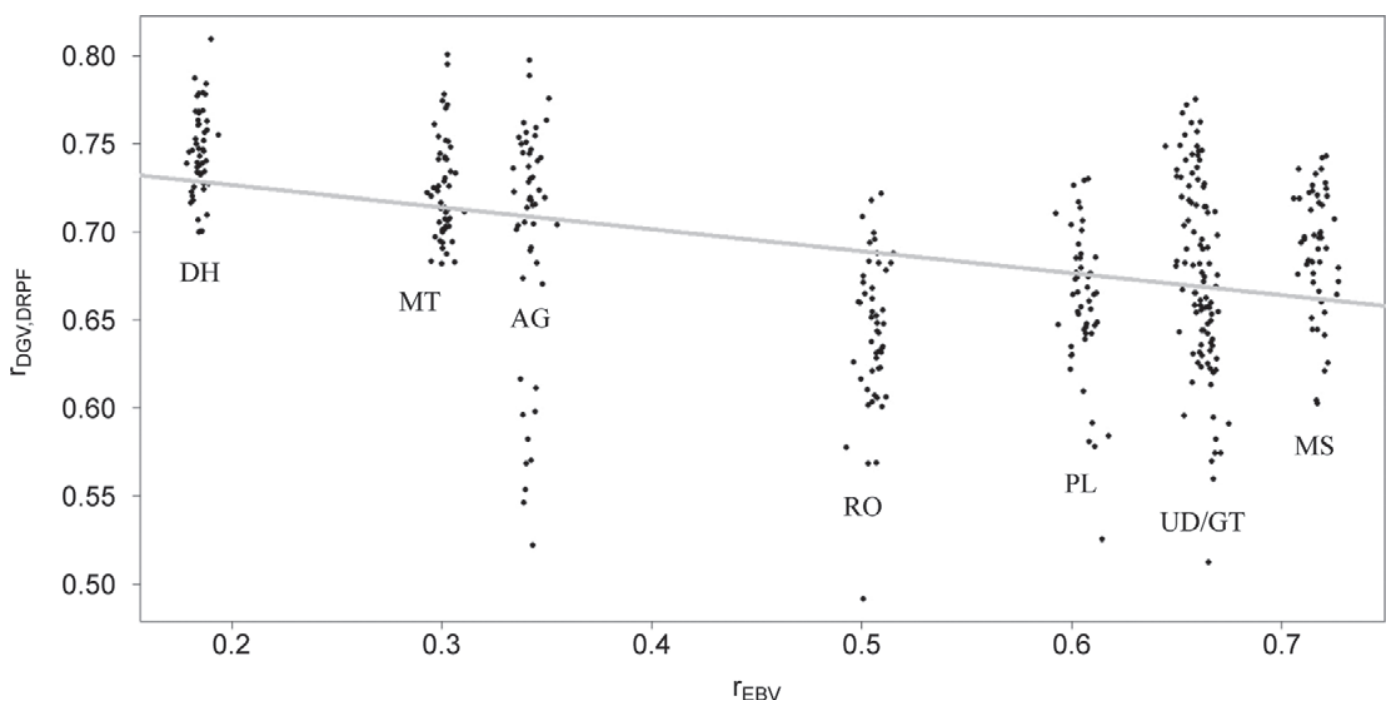

Figure 4. Linear regression of the correlation between direct genomic value and deregressed proofs $\left(r_{D G V . D R P F}\right)$ on the accuracy of EBV in the validation set $\left(r_{E B V}\right)$, simultaneously for all 8 traits: days to first heat $(\mathrm{DH})$, milking temperament (MT), aggressiveness (AG), rank order in herd (RO), position of labia (PL), udder depth (UD), general temperament (GT), and milking speed (MS).

$\left(a_{0}=0.75 \pm 0.03 ; a_{1}=-0.76 \pm 0.38\right.$, and $a_{2}=0.64 \pm$ $0.38)$. Thus, the advantage of this second option seems to be limited.

\section{Results for SNP Effects}

Genomic regions with clusters of SNP with large effects ( $>4$ SD above the mean) on the traits observed are listed in Table 3. When annotating these regions, we found 2 candidate genes that might influence the functional traits observed. The TAC1 (tachykinin, precursor 1) gene is located within a peak on chromosome 4 for general temperament. This gene encodes a member of the tachykinin peptide hormone family, which play a role in behavior response (Chiwakata et al., 1991) and might thus also affect the general temperament of an animal. For the trait days to first heat, we found a peak in the center of chromosome 21. Four genes are located here, one of which is $C Y P 11 A 1$, which encodes the cholesterol side-chain cleavage enzyme.

Table 3. Localization of regions with possible high influence on the observed traits, denomination of SNP with the greatest effect within that region (SNP ID), and genes up to $100 \mathrm{~kb}$ upstream and downstream of the SNP with the greatest effect within that region

\begin{tabular}{|c|c|c|c|c|}
\hline Trait & SNP ID & BTA & Position (bp) & Gene $100 \mathrm{~kb}$ upstream or downstream ${ }^{1}$ \\
\hline \multirow[t]{2}{*}{ General temperament } & BovineHD0400004398 & 4 & $14,887,518$ & $T A C 1$ \\
\hline & BovineHD0800030109 & 8 & $101,460,591$ & $A K A P \mathscr{Q}, T X N, T X N D C 8$ \\
\hline Milking temperament & BovineHD1400015525 & 14 & $55,580,399$ & \\
\hline \multirow[t]{2}{*}{ Aggressiveness } & BovineHD0800017506 & 8 & $58,503,427$ & \\
\hline & BovineHD2000018921 & 20 & $65,702,103$ & $A D C Y 2$ \\
\hline \multirow[t]{4}{*}{ Rank order in herd } & BovineHD0100035735 & 1 & $126,516,898$ & SLC9A9 \\
\hline & BovineHD0600001952 & 6 & $8,448,579$ & \\
\hline & BovineHD1800009080 & 18 & $29,383,349$ & \\
\hline & BovineHD1900003727 & 19 & $14,062,783$ & $A C A C A, T A D A 2 A, D U S P 14, S Y N R G$ \\
\hline \multirow[t]{5}{*}{ Milking speed } & BovineHD0500031516 & 5 & $109,364,642$ & $C A C N A 1 C, I L 17 R A, C E C R 5, C E C R 1$ \\
\hline & BovineHD0900004541 & 9 & $16,815,501$ & \\
\hline & BovineHD1100024011 & 11 & $83,456,178$ & \\
\hline & BovineHD1200003011 & 12 & $10,668,524$ & $O L F M 4$ \\
\hline & BovineHD1400018332 & 14 & $65,573,091$ & $Y W H A Z$ \\
\hline \multirow[t]{2}{*}{ Udder depth } & BovineHD0300020780 & 3 & $70,574,840$ & TNNI3K \\
\hline & ARSBFGLNGS13749 & 6 & $67,877,316$ & ATP10D, CORIN \\
\hline \multirow[t]{2}{*}{ Position of labia } & BovineHD0800030481 & 8 & $102,549,325$ & $Z N F 483$ \\
\hline & BovineHD2500002490 & 25 & $9,358,007$ & ATF'IP2, EMP2, TEKT5 \\
\hline \multirow[t]{3}{*}{ Days to first heat } & BovineHD0400024614 & 4 & $88,917,242$ & $W A S L, S P A M 1$ \\
\hline & BovineHD1500021240 & 15 & $73,554,550$ & \\
\hline & BovineHD4100015166 & 21 & $34,675,608$ & UBL, SEMA \\
\hline
\end{tabular}

${ }^{1}$ Genes in bold face could affect the observed trait. 
This enzyme catalyzes the composition of pregnenolone from cholesterol, a steroid hormone involved in fertility (Heo et al., 2012). The CYP11A1 gene might influence the level of estrogen and thus the time span between parturition and occurrence of first heat.

\section{CONCLUSIONS}

As stated above, limited work has evaluated genomewide association of functional traits in Brown Swiss, especially for behavior traits or the new conformation trait position of labia. We found no biologically plausible candidate gene that might influence milking temperament, although we observed a distinct peak on chromosome 14. Larger studies and more collaboration between geneticists and molecular biologists are necessary to identify genes affecting the functional traits studied here.

\section{ACKNOWLEDGMENTS}

The authors gratefully acknowledge co-funding from the European Commission, under the Seventh Framework Program for Research and Technological Development, for the Collaborative Project LowInputBreeds (Grant agreement No 222623). However, the views expressed by the authors do not necessarily reflect the views of the European Commission, nor do they in any way anticipate the Commission's future policy in this area. Parts of this research were also funded by the German Federal Ministry of Education and Research $(\mathrm{BMBF})$ within the AgroClustEr "Synbreed - Synergistic plant and animal breeding" (FKZ 0315528C). The authors thank Braunvieh Schweiz (Zug, Switzerland), the genotype pool Germany-Austria, Associazione Nazionale Allevatori Bovini della Razza Bruna (Bussolengo, Italy), and Beltsville Agricultural Research Center (Beltsville, MD) for provision of genotypes.

\section{REFERENCES}

Amer, P. R., and G. Banos. 2010. Implications of avoiding overlap between training and testing data sets when evaluating genomic predictions of genetic merit. J. Dairy Sci. 93:3320-3330.

Chiwakata, C., B. Brackmann, N. Hunt, M. Davidoff, W. Schulze, and R. Ivell. 1991. Tachykinin (substance-P) gene expression in Leydig cells of the human and mouse testis. Endocrinology 128:24412448.

Edel, C., R. Emmerling, and K.-U. Götz. 2010. A modification of VanRaden's index for the blending of genomic breeding values. P0324 in Proc. 9th World Congr. Genet. Appl. Livest. Prod., Leipzig, Germany. German Society for Animal Science, Neustadt, Germany.

Edel, C., R. Emmerling, S. Neuner, and K. U. Götz. 2011. Genomische Selektion: Stand der Implementierung beim Fleckvieh. Zuchtungskunde 83:257-265.
Erbe, M., B. J. Hayes, L. K. Matukumalli, S. Goswami, P. J. Bowman, C. M. Reich, B. A. Mason, and M. E. Goddard. 2012. Improving accuracy of genomic predictions within and between dairy cattle breeds with imputed high-density single nucleotide polymorphism panels. J. Dairy Sci. 95:4114-4129.

Erbe, M., F. Seefried, and H. Simianer. 2011. Effect of relationship and age structure between training and validation set on the accuracy of genomic breeding value prediction using genomic BLUP. Adv. Anim. Breed. Genet. 19:287-290.

Garrick, D. J., J. F. Taylor, and R. L. Fernando. 2009. Deregressing estimated breeding values and weighting information for genomic regression analyses. Genet. Sel. Evol. 41:55.

Gilmour, A. R., B. J. Gogel, B. R. Cullis, and R. Thompson. 2009. ASReml User Guide. Release 3.0. VSN International Ltd., Hemel Hempstead, UK.

Gredler, B., M. Sargolzaei, B. Bapst, A. Bieber, H. Simianer, and F. R. Seefried. 2013. Accuracy of 54K to HD genotype imputation in Brown Swiss cattle. P0534 in Proc. Plant and Animal Genome XXI, San Diego, CA. Scherago International, Jersey City, NJ.

Guo, J., H. Jorjani, and Ö. Carlbord. 2012. A genome-wide association study using international breeding-evaluation data identifies major loci affecting production traits and statue in the Brown Swiss cattle breed. BMC Genet. 13:82. http://dx.doi.org/10.1186/14712156-13-82.

Heo, G. Y., W. L. Liao, I. V. Turko, and I. A. Pikuleva. 2012. Features of the retinal environment which affect the activities and product profile of cholesterol-metabolizing cytochromes P450 CYP27A1 and CYP11A1. Arch. Biochem. Biophys. 518:119-126.

Kramer, M., M. Erbe, B. Bapst, A. Bieber, and H. Simianer. 2013. Estimation of genetic parameters for novel functional traits in Brown Swiss cattle. J. Dairy Sci. 96:5954-5964.

Ober, U., F. Ayroles, E. A. Stone, S. Richards, D. Zhu, R. A. Gibbs, C. Stricker, D. Gianola, M. Schlather, T. F. C. Mackey, and H. Simianer. 2012. Using whole-genome sequence data to predict quantitative trait phenotypes in Drosophila melanogaster. PLoS Genet. 8:e1002685. http://dx.doi.org/10.1371/journal.pgen.1002685.

Saatchi, M., M. C. McClure, S. D. McKay, M. M. Rolf, J. W. Kim, J. E. Decker, T. M. Taxis, R. H. Chapple, H. R. Ramey, S. L. Northcutt, S. Bauck, B. Woodward, J. C. M. Dekkers, R. L. Fernando, R. D. Schnabel, D. J. Garrick, and J. F. Taylor. 2011. Accuracies of genomic breeding values in American Angus beef cattle using K-means clustering for cross-validation. Genet. Sel. Evol. 43:40.

Sargolzaei, M., J. P. Chesnais, and F. S. Schenkel. 2011. FImpute An efficient imputation algorithm for dairy cattle populations. J. Dairy Sci. 94(E-Suppl. 1):421. (Abstr.)

Segelke, D., J. Chen, Z. Liu, F. Reinhardt, G. Thaller, and R. Reents. 2012. Reliability of genomic prediction for German Holsteins using imputed genotypes from low-density chips. J. Dairy Sci. 95:5403-5411.

Su, G., R. F. Brøndum, P. Ma, B. Guldbrandtsen, G. P. Aamand, and M. S. Lund. 2012. Comparison of genomic predictions using medium-density $(\sim 54,000)$ and high-density $(\sim 777,000)$ single nucleotide polymorphism marker panels in Nordic Holstein and Red Dairy cattle populations. J. Dairy Sci. 95:4657-4665.

VanRaden, P. M. 2008. Efficient methods to compute genomic predictions. J. Dairy Sci. 91:4414-4423.

VanRaden, P. M., D. J. Null, M. Sargolzaei, G. R. Wiggans, M. E. Tooker, J. B. Cole, T. S. Sonstegard, E. E. Connor, M. Winters, J. B. C. H. M. van Kaam, A. Valentini, B. J. Doormaal, M. A. Faust, and G. A. Doak. 2013. Genomic imputation and evaluation using high-density Holstein genotypes. J. Dairy Sci. 96:668-678.

Wellmann, R., S. Preuß, E. Tholen, J. Heinkel, K. Wimmers, and J. Bennewitz. 2013. Genomic selection using low density marker panels with application to sire line pig breeding. Genet. Sel. Evol. $45: 28$. 